\title{
A Novel Method for Higher Order Aberration Correction in Electron Microscopes
}

\author{
Shahedul Hoque ${ }^{1,2}$, Hiroyuki Ito ${ }^{1}$, Akio Takaoka ${ }^{2}$ and Ryuji Nishi $^{2}$ \\ 1. Hitachi High-Technologies Corp., Ibaraki, Japan. \\ 2. Osaka University, Osaka, Japan.
}

Aberration correction is an important technology for improving resolution of electron microscopes, having wide applications in the fields of nanotechnology and material science. Correctors for 3rd order spherical aberration $\left(C_{S}\right)$ have shown significant improvement of resolution. For higher S/N imaging by increasing the probe current, and for improvement of resolution by suppressing diffraction effect especially at lower beam energy, aperture angle becomes larger and hence higher order aberrations also need to be corrected. Again, it is extremely complicated to control conventional correctors, because they are made of magnetic material, which suffers from hysteresis, inhomogeneity, magnetic saturation and susceptibility to noises. In this paper, we present a novel and simple method for correcting higher order aberrations. To overcome the drawbacks of conventional correctors, we utilize a setup called $\underline{N}$-fold symmetric line currents ( $N$-SYLC).

$N$-SYLC is a setup of electric currents parallel to the optic axis of microscope with $N$-fold symmetry. It generates $2 \mathrm{~N}$-pole field in the proximity of optic axis according to the Biot and Savart Law, without using any magnetic material [1]. For example, for $N=3$ it generates sextupole field and is denoted as 3SYLC. N-SYLC can be realized by toroidal coils. Figure 1 shows schematic of 3-SYLC.

We recently showed that 3-SYLC doublet can work as a $C_{s}$ corrector [2]. In this paper, we show that after $C_{s}$ correction, the most dominant higher order aberrations are two 5th order aberrations, namely, 5 th order spherical aberration $\left(C_{5}\right)$ and sixfold symmetric astigmatism $\left(A_{5}\right)$. We analytically demonstrate that $C_{5}$ can be corrected by superposition of octupole field $(N=4)$, and $A_{5}$ by dodecapole field $(N=6)$. Thus, the corrector should be a combination of 3-SYLC, 4-SYLC and 6-SYLC, which we denote compactly as $2 \times(3+4+6)-S Y L C$.

We find that superposition of sextupole and octupole fields results in new aberrations which should also be cancelled. We prove analytically that by using quadruplet or four $2 \times(3+4+6)$-SYLC arranged along the optic axis, each being connected to the next by a pair of transfer lenses (see Figure 2 for schematic), and by imposing certain symmetry conditions on the coil currents, all these aberrations can be cancelled.

The above analysis is based on simplified models ignoring the detailed structure of $N$-SYLC. To consider a realistic case, we also perform computer simulations with toroidal coils as $N$-SYLC. We use a software called the LANTERN developed by MEBS [3], which computes the electron trajectories by direct ray tracing, including aberrations of all order. We consider low energy beam of $5 \mathrm{keV}$, since larger aperture angle is needed to suppress diffraction effect at low energy. Figure 3 shows beam cross section at the corrector image plane for initial angle $10 \mathrm{mrad}$. If the magnification of objective lens is $M$, the aperture angle at the specimen plane is $10 / M$ mrad. After $C_{s}$ correction beam size is determined by higher order aberrations. $C_{5}$ and $A_{5}$ are corrected simultaneously and independently by our model, and the final beam size is less than $0.5 \mathrm{~nm}$ at the corrector image plane. 
Thus, $2 \times(3+4+6)$-SYLC quadruplet with specific symmetry conditions presents a realistic model for higher order aberration correction in electron microscopes. Absence of magnetic materials should make it easily controllable. All axial geometrical aberrations are corrected up to and including 5th order to yield sub-nm resolution even at large aperture angles.

\section{References:}

[1] R. Nishi, H. Ito, S. Hoque, $18^{\text {th }}$ International Microscopy Congress (IMC2014), Prague, Czech Republic, (2014), p. 200-201.

[2] S. Hoque, H. Ito, R. Nishi, A. Takaoka and E. Munro, Ultramicroscopy 161 (2016), p. 74-82.

[3] Munro's Electron Beam Software Ltd., http://www.mebs.co.uk/

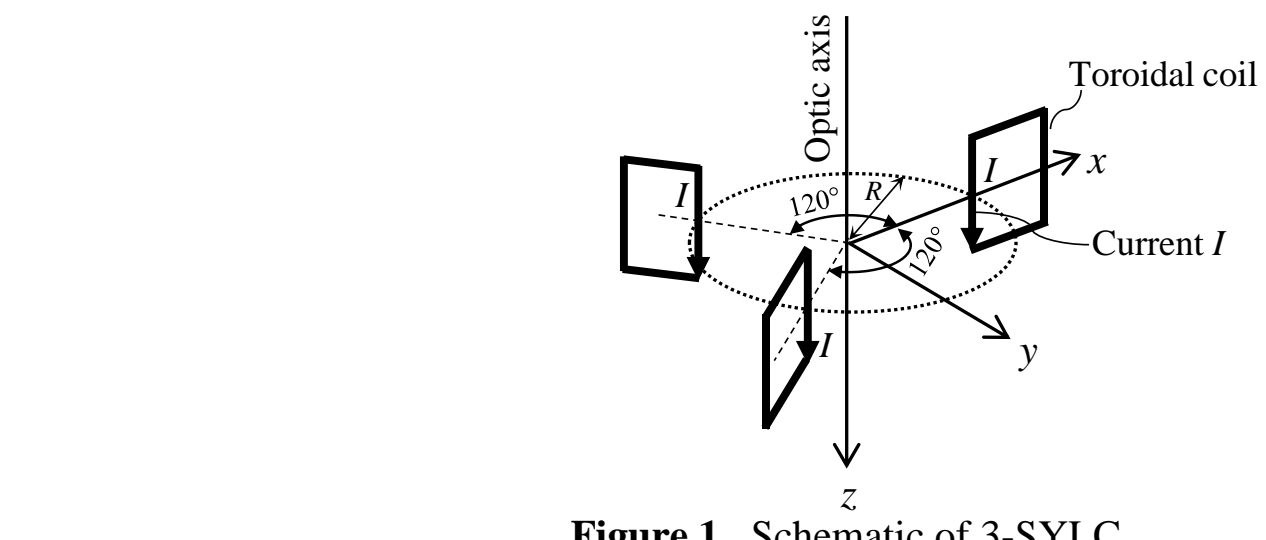

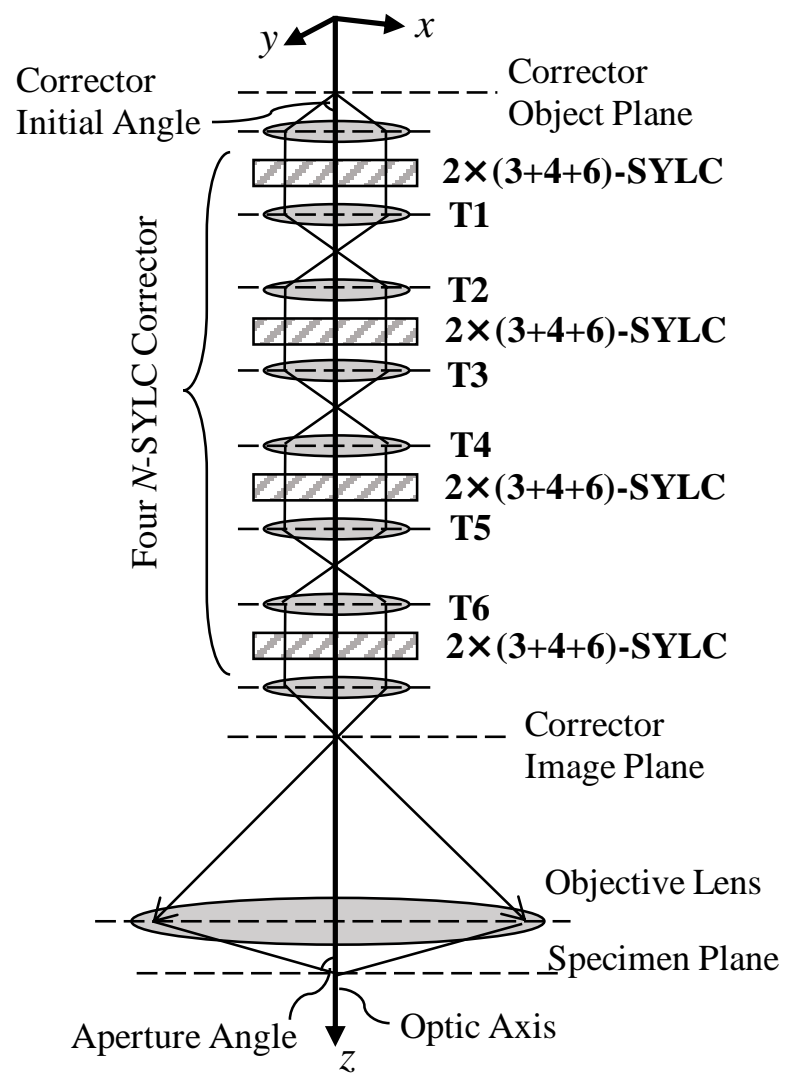

Figure 2. Arranging four $2 \times(3+4+6)$-SYLC along the optic axis. T1 to T6 are transfer lenses.

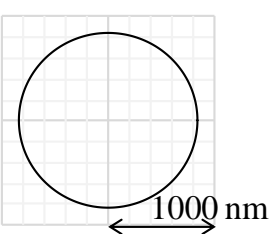

Before aberration correction

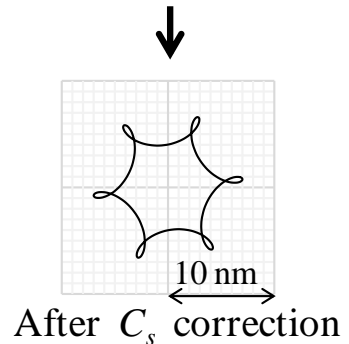

$\downarrow$

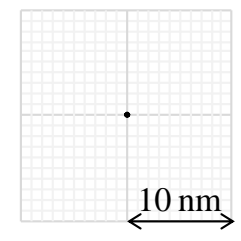

After 5th order aberration correction

Figure 3. Beam cross section at the corrector image plane before and after aberration correction 